\title{
Téoros
}

Revue de recherche en tourisme

\section{Le parc national des Abruzzes ou un mariage réussi entre environnement et développement}

\section{Aimé Mucci}

Volume 14, numéro 1, printemps 1995

Les parcs : des réseaux en mutation

URI : https://id.erudit.org/iderudit/1077060ar

DOI : https://doi.org/10.7202/1077060ar

Aller au sommaire du numéro

Éditeur(s)

Université du Québec à Montréal

ISSN

0712-8657 (imprimé)

1923-2705 (numérique)

Découvrir la revue

Citer cet article

Mucci, A. (1995). Le parc national des Abruzzes ou un mariage réussi entre environnement et développement. Téoros, 14(1), 55-59.

https://doi.org/10.7202/1077060ar d'utilisation que vous pouvez consulter en ligne.

https://apropos.erudit.org/fr/usagers/politique-dutilisation/ 


\section{Le parc national des Abruzzes ou un mariage réussi entre environnement et développement}

Aimé Mucci

À la fin du deuxième millénaire, l'humanité s'interroge sur le destin du monde. L'Italie, avec $4 \%$ de son territoire d'aires protégées, ne se situe quà la $17^{\circ}$ place des 24 pays européens qui veillent à la protection de leur environnement. Après trente années d'attente et de débats passionnés, elle s'est enfin dotée d'une loi-cadre concernant les zones sauvegardées. Cette loi, entrée en vigueur en 1991, est porteuse d'un soénario plus vaste et prometteur pour ce pays.

Les actions vigoureuses des Associations des Amis de la nature ont réussi à faire admettre que l'Italie devienne enfin une «terre de parcsverts»entourée d'une «mer de parcs bleus* (carte).

L'idée désintéressée de la conservation du patrimoine représente une des plus belles conquêtes de notre civilisation moderne.

Le droit à l'environnement fait partie de nos droits fondamentaux et sa protection, écrivait en 1971 le professeur Franco Tassi ${ }^{(2)}$, se place sur le même plan que celle de la vie.

\section{L'environnement est un droit et un devoir}

Le milieu où nous vivons, qui ne doit pas être considéré seulement sous l'aspect esthétique, mais comme une matière vivante faite d'animaux, de plantes, d'eaux et de roches, est un bien collectifettout le monde a ledroitd'en jouir. Chaque bénéficiaire de ce «don du ciel» doit pouvoir le défendreet le gérer dans son propre intérêt, mais aussi dans celui des générations futures.

Dans nos pays fortement industrialisés, il n'est pas possible de considérer l'homme isolément et de ne tenir compte que de la communauté humaine. Il est indispensable d'englober dans nos considérations l'ensemble des êtres vivants. Par ailleurs, le

Monsieur Aimé Mucci est professeur il l'Unwer sité Toulouse-Le Mirail et directeur de l'équipe de recherche Tourisme Socal. milieu n'étant pas un système ouvert d'où il est possible d'extraire indéfiniment des ressourceset auquel il est toléré de restituer les déchets, l'environnement ne supporte pas l'exploitation et la dégradation au-delà de certaines limites.

Chacun de nous doit prendre conscience que le milieu, en plus d'être un bien en soi, est également un bien économique qui mérite d'être préservé. Il nes'agit pas d'un objet quelconque dont on peut jouir et que l'on peut échanger ou même détruire; c'est un bien devaleur qu'il fautgérer etconserver dans l'intérêt du présent, mais surtout de l'avenir.

Dans cette prise de conscience, l'Italieavait un énorme retard. Les gens de ce pays, qui bénéficient pourtant d'une nature généreuse, se sont longtemps désintéressés de ce bien si précieux. Jusqu"à ces dernières années, le législateur italien n'avait pas pris en considération les exigences de conservation, de sauvegarde et d'utilisation du milieu naturel. Le sol était victime d'une quantité d'abus, les déchets étaient abandonnés sans tenir compte de la dégradation etde la pollution des sites, lesdéboisements excessifs et inconsidérés provoquaient et provoquent encore ${ }^{(3)}$ éboulements et inondations. Tousles Partis, par crainte de l'impopularitéet victimesd'un dientélisme viscéral, ne se rendaient pas compte que la sauvegarde de l'environnement, sa valorisation et toutes les mesures aptes à favoriser la jouissance rationnelle de ce bien de la part de la collectivité représentent un service social fondamental. L'Italie possédait des Pares naturels mais les défenseurs du milieu eurent du mal à démontrer la nécessité et la validité des parcs nationa ux et à faire admettre leur idée.

\section{Qu'est-ce qu'un Parc National?}

C'est avant tout un bien où la nature est protégée, un bien qui n'est pas l'objet d'interventions, de transformations de la part de l'homme. Dans un Parc National, on n'abat pas la forêt, on ne construit pas, on ne pollue pas; les animaux et les plantes se reproduisent en totale liberté. On peut certes y accéder, mais en prenant des précautions précises.

Même si elle est difficilement quantifiable, l'utilité d'un Parc est évidente et joue plu= sieurs rôles: culturel, éducatif, récréatif, social et touristique. Culturel puisqu'il permet de mieux connaître la nature et toutes les problématiques liées au milieu: flore, faune, géomorphologie, traditions, histoire, légendes liées à la civilisation des bergers. Le Parc, et ce sera le cas pour celui des Abruzzes, occupant une position charnière entre le Nord et leSud del'Italie, constitue une sorte d'artère fondamentale d'échange de cultures différentes. Il permet de parcourir des itinéraires au coeur de la nature à pied, à cheval, à VTT, à ski de fond mais favorise aussi des séjours scolaires avec des guides spécialisés et la collaboration de l'Association des hồteliers et restaurateurs qui font revivre les anciennes traditions culinaires. En favorisant la recherche pure et appliquée, le Parc joue un rôle formateur et informateur pour les jeunes générations, il constitue un véritable laboratoire scientifique universel en plein air. Le Parc a en quelque sorte une action sociale puisqu'il permet une saine utilisation du temps libre et a des bienfaits psychologiques ethygiéniques. Il revêtaussi une valeur urbanistique, puisqu'il donne la possibilitéà de vieux villages de revivre tout en conservant leur aspect d'antan. La nature et ses habitants ne sont pas comme lespalais détruits par la guerre. Ces derniers peuvent être reconstruits, mais si la nature est anéantie, personne ne pourra la faire revivre.

\section{Le Parc national des Abruzzes: le résultat d'un long combat}

A l'origine, la zone qui aujourd'hui est devenue un Parc National n'était qu'une réserve royale ayant pour but de préserver les espèces rares. L'idéedu Parcfutproposée en 1907 par la société émilienne Promomtiluss $e$ Silvis et ne comprenait que 500 hectares. Puis grâce à l'action d'Erunnio Spari, un défenseur acharné de la nature, la réserve initiale est transformée en Parc National des Abruzzes par décret-loi du 11 janvier 


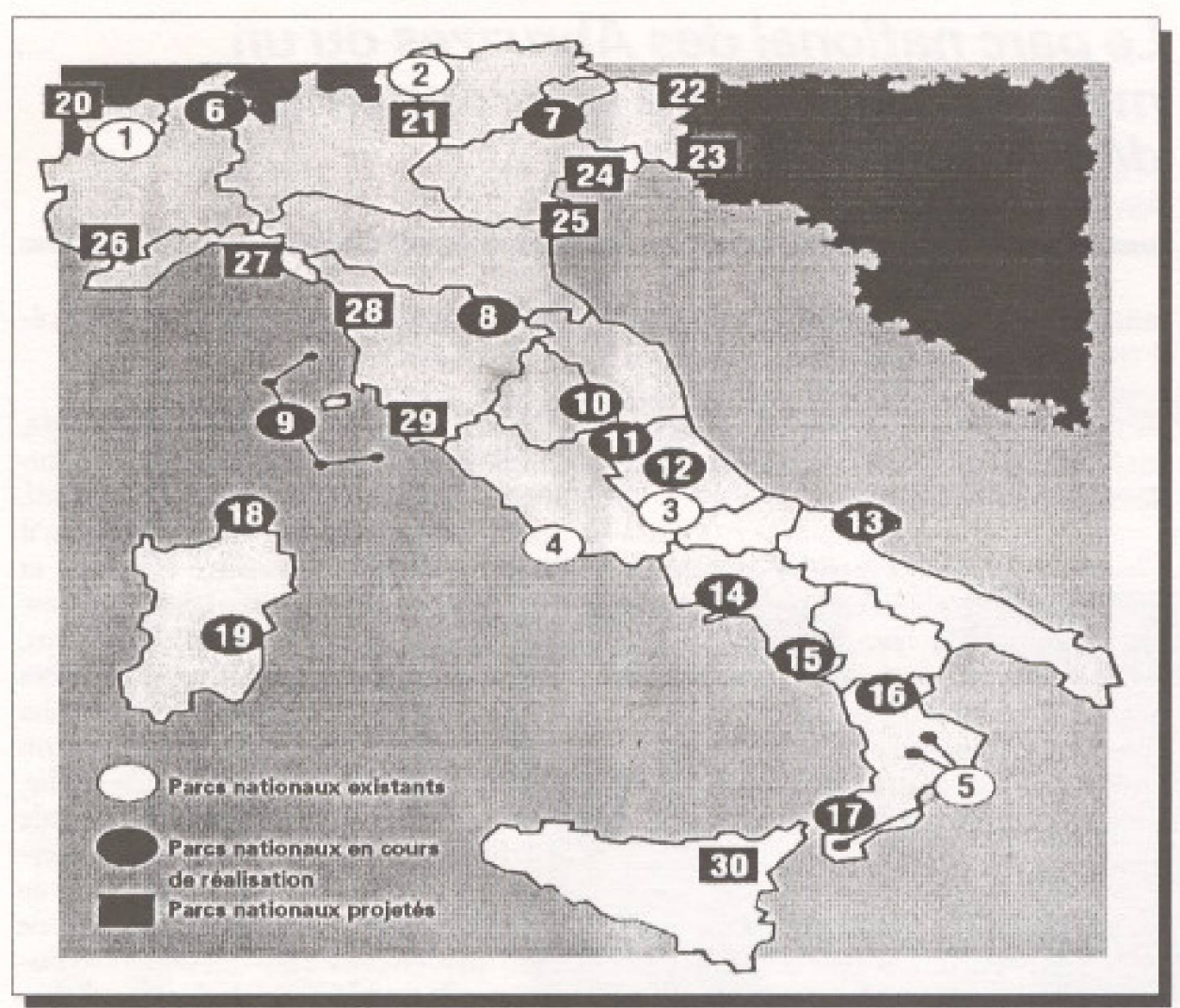

1923 et sa superficie est alors de 18000 hectares. Celle-ci sera portée à 28000 hectares en 1925 et à 30000 en 1926 . En 1936, le Parc est supprimé en tant que tel et la gestion du territoire qu'il couvrait est confiée à la Milice forestière. En 1950, un Office autonome est reconstitué mais, en 1953 il est l'objet d'une nouvelle menace avec la spéculation foncière et les investisseurs immobiliers. Un dixième de la super ficiedu parc, soit 3000 hectares, est détruit. Ce n'est qu'en 1970 que l'opinion publique est alertée par la presse et les milieux culturels. Une meilleure planification est apportée à l'environnement. En 1972, la Communauté Européenne reconnaît l'importance de l'aire protégéedes Abruzzes et lui décerne un diplôme européen. En 1975, sur initiative de la Région Abruzzes, il est procédéà un jumelage avec la forêt bavaroise. L'année suivante l'aire s'approprie le massif du Mont Morsicano soit 10000 hectares. En 1990 , on y ajoute le Manarde, soit 4000 hectares. Ce qui porte l'ensemble à 44000 hectares protégés directement. A ce territoire s'ajoutent 60000 hectares deprotection externegrâce à l'intéressement des Régions du Latium et du Molise. Dess lors, les activités humaines exercées sur le Parc sont contrôlées, des règlements précis disciplinent le flux tou- ristique et la nature, sous toutes ses formes, est respectée et protégée.

Sur la zone, dite intégrale, il est interdit de sortir des sentiers balisés et il faut être accompagnéd'un personnel spécialisé, mais des autorisations sont accordées pour la recherche scientifique. Toutes les altérations du milieu sont sévèrement réprimandees.

\section{Le Parc National des Abruzzes : une réalisation significative}

Cette aire protégée au centre de l'Italie concerne 3 régions: Abruzzes, Latiom et Molse, 3 provinces, 22 communes (dont 5 à l'intérieur du Parc) et 6 communautés de montagne. Dans cet ensemble, nous pouvons relever 1200 essences d'arbres, 40 espèces de mammiferes, 300 d'oiseaux, 30 de reptiles ou amphibies, de 70 à 100 ours bruns des marriche, 35 à 40 loups, 450 à 500 chamois d'Abruzzes, 500 à 600 cerfs, 150 à 200 chevreuils. Il a été procédé à une structuration du territoire avec des zones à régime différencié comportant un Plan pour le Parc proprement dit et un Plan d'urbanisme. Il s'agit de deux instruments distincts complémentaires et essentiels; chacun a des perspectives propres, des priorités politiques, des bases réglementai- res et des échelles de mesure différentes. L'ensemble comporte quatre zones. La zone A couvre $10 \%$ à $20 \%$ du territoire et constitue une réserve intégrale avec une conservation absolue. Aucune altération ne doit être apportée à tout ce qui représente la vie, sauf pour certaines mesures de sauvegarde. Lesvisiteursdoiventsedéplacer uniquement à pied le long d'itinéraires obligés.

La zone B avec $60 \%$ à $70 \%$ de la superficie totale constitue une réserve générale bien conservée. La présence humaine séculaire y est maintenue. Une évolution socioéconomique est favorisée en procédant à une reconversion graduelle. Les activités agropastorales et la pëche y sont tolérées, mais sous le contrôle des autorités du Parc. Les bâtiments existants sont conservés et restaurés. Sans autorisation préalable, aucune initiative nouvelle n'est tolérée. Les visiteurs peuvent s'y déplacer à pied, à cheval ou à ski sur des iunéraires préférentiels mais libres. Quant aux engins motorisés, ils ne sont autorisés que sur le réseau routier défini par l'autorité responsable du Parc. La zone C dite de protection concerne $20 \%$ a $25 \%$ de l'aire. Les activités économiques traditionnelles y sont permises; elles peuvent être étendues ou reconverties à condition de respecter les coutumes de la région. La zone D permet le développement; elles'étend sur $5 \%$ à 10 $\%$ de la superficie totale. C'est là que se trouvent les réalisations nécessaires à la vie et au développement des activités économiques avecla réalisation des infrastructures réceptives indispensables ainsi que les equipements correspondants.

Pour mener à bien cette action de grande ampleur, la participation de la population locale à la gestion des aires protégées est indispensable.

Lespersonnesvivantsur placeneserendent pas toujours compte du caractère exceptionnel de leur territoire. Même si elles prennent conscience du problème, elles interviennent trop tard et souvent les collectivités locales ne sont pas en mesure d'evaluer les difficultés et d'y faire face. Aussi une autorité centrales'est-elle avérée indispensable, car le Parc a besoin d'une protection directe et d'une protection indirecte. L'autorité compétente a établi un plan clair et un règlement; c'est elle qui rembourse les dégâts éventuellement causés aux troupeaux par les loups, essaie de résoudre le problème des chiens errants et 
veille à ce que le nombre de visiteurs ne dépasse pas le seuil toléré. Conscients du fait que le Parc est une epoule aux oeufs d'ors qu'il ne faut ni affaiblir ni tuer, les responsables doiventrésister aux waffairistes du verDs, aux appétits individuels sectoriels ou locaux et lutter contre l'égoïsme myope. L'autorité centrale joue alors le rôle de médiateur face aux intérêts en jeu. Responsable de la promotion culturelle et économique des zones protégées, elle permet aux collectivités locales d'être les véritables protagonistes.

Pour permettre le développement des services de base du Parc, toutes les tâches commerciales et productives sont décentralisées et déléguées à des organisations locales prévues à cet effet. Des associations de volontaires et des coopératives opèrent sous le contrôle de l'autoritédu Parc; celleci collabore étroitement avec les Régions, les Communes, les Communautés de montagne concernées. Des organisations privées, syndicales ou professionnelles ainsi que des administrations publiques centrales et locales participenta la lagestion du Parc et à son évolution. Les difficultés sont nombreuses, car il s'agit d'une problématique qui englobe une série d'intérêts économiques, politiques et scientifiques. L'absence d'une loi cadre (elle ne sera adoptée qu'en 1991) fut un obstacle important à l'extension du Parc quidonna lieu à de nombreuses polémiques entre les amis du Parc et les partisans du laisser-aller.

\section{L'extension du Parc et les luttes qu'elle provoque}

Si un Office autonome est reconstitué en 1950, ce n'est qu'en 1976 que se réalise le souhait des scientifiques, des associations culturelles et protectrices de la nature et d'une parte importante de l'opinion publique et étrangère avec l'extension de 10 000 hectares supplémentaires, grâce à l'insertion du massif Marsicano.

Cette décision prise par le ministère de l'Agriculture donna lieu à une âpre bataille juridique et à de nombreuses campagnes de presse en raison de l'importance des intérêts économiques en jeu. Le procès est intenté par la région des Abruzzes ellemême et par trois des 18 communes impliquées dans le Parc dont Pesanserdi (qui occupe une position centrale etoù se trouve actuellement la direction du Parc), Opi (centre du chamois) et Scamo.
La première phase administrative se termine en faveur du Parc par décision du TAR Abruzzes (Tribunal Administratif Régional) en date du 8 novembre 1978 et l'action est ensuite engagée, le 4 septembre 1979, devant le Conseil d'Etat qui rejette la plainte.

Leparadoxeest dû au fait queles adversaires les plus acharnés du Parc ne sont pas des propriétaires privés, mais bien les organismes publics territoriaux avec, à leur tếte, la Région Abruzzes. Il ne s'agit pas d'une question de procédure comme les plaignants voudraient le faire accroire mais tout simplement parce que les parties hostiles, y compris les responsables des collectivités locales, avaient souscrit des documentsofficielsavecdes sociétés privées extérieures qui souhaitaient implanter des stations de ski, des immeubles et des résidences secondaires. Ce sont donc des coulées de béton pour une réalisation massive de routes, d'installations sportives et de complexes résidentiels, une sorte de colonisation avec des capitaux étrangers sans aucune participation effective du tissu social qui menaçaient ces contrées si belles. La Commune de Pecasserali par exemple, dans sa délibération $n^{\circ} 23$ du 24 février 1975 avait accordé à la société Sisipma la concession de 9 implantationsde ski et tous les ouvrages nécessaires à leur bon fonctionnement sur le mont Marsicana. En cette circonstance, la presse qui s'était montrée hostile au Parc et qui annonçait unecatastrophe économique pour la Région ne fit paspreuve de clairvoyance puisqu'elle ne se rendait pascomptequ'une valorisation touristique faite de résidences secondaires, de télésièges et d'équipements pour des gens qui ne seraient venus que pendant quelques jours et quelques semaines d'été ou d'hiver ne pouvait être qu'éphémère. On ne peut pas construire à l'infini sans détruire l'environnement et l'on ne peut pasassurer aux populations locales unavenir valable après une période d'emploi sur les chantiers de construction. Il ne faut pas voir un conflit entre urbanisme et défense de la nature, mais voir dans la nature la limite nécessaire pour toute intervention sur le territoire. L'agrandissement d'un parc naturel va vraiment dans le sens des exigences fondamentales de l'urbanisme et de la discipline du territoire parce qu'il compense les graves déséquilibres du développement industriel et de la croissancedes villes.
De nombreuses années passèrent avant que la question ne revienne d'actualité et cela grâce aux progrès réalisés par le Parc des Âbruzzes d'importance nationale. $\mathrm{Ce}$ n'est qu'en 1989 que quelques maires ayant constaté la renaissance des villages du Parc et en particulier de Civitella Alfedena aidés par la volonté des écologistes de la Région Moliseetdes WWF se firent les défenseurs de l'environnement. C'est alors que le Président de la République signe, le 10 janvier 1990 , le décret concernant le Projet Mainarde qui ajoute 4000 hectares au parc portant ainsi sa superficie à 44000 hectares. Il fallut alors intégrer ce territoire magnifiqueavectoutson patrimoine naturel mais aussi historique, humain et culturel dans la réalité vivante et dynamique du Parc; garantir la conservation de ses valeurs fondamentales en assurant un développement raisonnable et harmonieux de ces villages condamnés à voir leurs habitants émigrer ou pire encore à l'invasion par le tourisme de masse. Les défenseurs du Parc se sont mis au travail pour récupérer et revitaliser des centres historiques et développer les forces endogènes locales.

\section{Une initiative surprenante et heureuse: I'opération Saint- François is}

Alors que la bataille au sujet de l'extension du Parc battait son plein, commençait celle pour la sauvegarde du loup des Apennins qui sous de nombreux aspects sera exemplaire puisqu'elle concerne l'un des sujets lesplus brûlants et le plusdifficile: la défense du prédateur «nocif, carnivore, impitoyable et méchantss sur lequel on a écrit et bâti les légendes les plus extraordinaires. II touche les cordes les plus sensibles, enflamme-des passions violentes et contrastées. La campagne pour le loup lancée en Italie voici plus de vingt ans, au-delà des brillants résultats obtenus, constitue un modèled'avant-garded'action intégréesur le plan de l'écosociologie. Pendant l'année 1971, au comble des persécutions dont est victimecettemalheureuse créaturevivante, il semblait impensable, non seulement de réussir la protection du loup, mais même d'avancer une proposition de ce genre.

L'opération Saint-François, exemple heureux de coopération entre le Parc National des Abruzzes et WWF, naquit dans ce climat. Bientôt la lutte éclata au milieu de difficultés presqueinsurmontables. On alla jusqu'à prétendre que l'on pratiquait un lancer aérien deloupscanadiens et sibériens. 
L'Italie se trouva coupée en deux et opposant deux cultures. D'un oôté la société traditionnelle basée sur l'élevage des brebis, mystiquement craintive à l'égard du loup et opposée pour des motifs évidents, parfois compréhensibles, mais presque toujours exagérés. De l'autre, la société moderne, urbaine, post-industrielle fascinée par ce achampion extrêmes du monde sauvage, rebelleirréductibleà la banalisation de l'homme, au point d'en faire un véritable symbole dans lequel projeter le désir croissant du retour à la nature, dans la lutte pour la sauvegarde des lieux encore non contaminés et des dernières créatures de la terre. Au début de l'opération, la lutte au loup se faisait dans un climat de chasse aux sorcières. Des battues publiques étaient organisées à la tête desquelles se trouvaient les forces del'ordre. Les loups «méchants», comprises les louves pleines et les louveteaux, étaient exterminés sans pitié à l'aide du fusil, des pièges ou des viandes empoisonnées. Les «vainqueurs passicient pour des héros et recevaient des prix en argent ou des prix en nature. Il fallait donc tenter de réhabiliter l'image de cet animal en détruisant les anciens mythes enracinés dans l'inconscient enfantin. Les dégâts imputés au loup dewaient être ramenés à leur juste valeur. Il fallait faire justice des croyances fausses, démontrer que le loup n'est pas agressif à l"égard de l'homme et démontrer son rôle dans l'équilibre écologique et dans le fonctionnement correct de lécosystème tout entier. Il ne s'agissait pas de recensement, d'études et de recherches, mais d'interventions intégrées à divers niveaux interdire avant tout l'utilisation des poisons et des pièges et indemniser les bergers des dommages subis par leurs troupeaux, et, en même temps, promouvoir des campagnes de sensibilisation et d'éducation. C'est ainsi qu'en 1974 naquit le Gruppo Lupo Italia, dans le cadre d'une vaste action internationale entreprise par Wolf del'IUCN (aujourd'hui Union Mondiale pour la Nature) dont le Président, Professeur H. Punlot, participa à un séminaire pour la protection du loup en Italie, qui s'est tenu le 20 juin 1973 dans le Parc des Abruzzes.

Pour atteindre un plein succès et pénétrer dans la citadelle fermée des mentalités locales, il fallait non seulement montrer la beauté du loup mais prouver son utilité. Dans ce but, à Cnnitella Alfedana a été créé un centre de visite entièrement consacré à cet animal. Grâce aux expositions didactiques, mais surtout grâce à l'observation directe du troupeau de loups qui depuis des années vivent et se reproduisent dans l'aire qui leur est destinée, toute proche, la légende du aloup méchant» est morte définitivement. Il $y$ a vingt ans, une empreinte de l'animal découverte hors du village suffisait pour faire verrouiller toutes les maisons. Le lendemain on pouvait lire sur les journaux: « $\mathrm{U}_{n}$ village sur les montagnes des Abruzzes assiegé par les loups». Aujourd'hui quand un troupea de loups en liberté s'approche des habitations, c'est la fête de la curiosité. Les vieux semontrent lespectacle inhabituel, tandis queles enfants ont si bien appris à imiter le cri de ces animaux qu'ils obtiennent des réponses fréquentessoit à l'intérieur de l'aire soit des montagnes voisines. Si dans de nombreuses zones de l'Apennin la pénurie de ressources peut transformer le loup en agresseur de troupeaux, dans le Parc des Abruzzes, la situation a évolué bien différemment grâce à la réintégration du cerf et du chevreuil réalisée ces dernières anneses avec un total succes. Alors qu'un loup seul n'est pas capable d'avoir raison d'un gros cerf, en réunissant ses forces, un troupeau de loups, peut réussir à s'emparer de quelques exemplaires plus fiibles, vieux ou malades, au nom de ce jeu cruel, mais nécessaire qu'est la sélection naturelle. En somme apress de nombreuses querelles et discussions, l'animal le plus significatif de notre culture passée et des anciennes traditions des Apennins a échappé à l'élimination et peut-être réussira-t-il h̀conserver sa place de créature sauvage.

Cette partie des Apennins, qui grâce au Parc National a pu réaliser une attraction culturelle et naturelle, a réussi à transformer des régions abandonnées, marginales et pauvres, en un «cas pilotes de développement.

\section{La protection de l'environnement et le développement économique}

Pendant les années 1950et 1960, les villages présents dans le Parc connaissaient les taux les plus élevés d'émigration de la péninsule italienne. Le développement lié au tourisme, en réalisant une attraction culturelle et naturelle, a apporté un fluxde ressources dans ces villages voués à l'abandon. Une nouvelle économie basée sur la communauté locale est née. Avec le Parc des Abruzzes est démontrée la thèse qui veut que même la nature peut offrir des bénéfices consistants a condition que l'on comprenne que ceux-ci ne jaillissent pas de sa consommation, mais en la laissant intacte pour en jouir aujourd'hui et demain par les générationsàvenir. Uninvestissement dans la nature est largement compensé par des bénéfices économiques durables et consistants.

Pour le Professeur Franco Tassi qui a consacré de nombreuses années de sa vieau Pare National des Abruzzes dont il était le directeur, «un Parc bien délimité et bien géré peut constituer la meilleure forme d'investissement pour un territoire et pour les communautés qui y vivent. Dans celui des Abruzzes 5 milliards de lires bien investis chaque année ont un impact économique de 300 milliards de lires, c"est-à-dire un rapport de 1 à 60 (dans l'industrie automobile ce rapport est de 1 à 4) $\%$. Ceci est d'autant plusimportantsil'on tient compte du fait que l'on ne détruit pas le patrimoine nature, mais bien au contraire que l'on favorise sa conserwation intelligente. Se développent alors toutes les activités liées à l'accueil et à la visite ainsi qu'un tertiaire avancé typique de l'écotourisme le plus moderne. En réalité, ce sont toutes les activités agropastorales qui sont vivifiées. Si le Parc donne du travail directement à une centaine de personnes et autant indirectement, l'effet induit est au moins dix fois supérieur. D'après une enquête de Nonismd ${ }^{(0)}, 1600$ petites entreprises familiales représentent la fòroe de base économique du Parc. Quant à l'écotourisme, il est destiné à croitre toujours plus. Le nombre de visiteurs s'élève à près de 2 millions par an. Or, il ne s'agit pas seulement de tourisme traditionnel ou de masse concentré sur un mois ou deux par an, mais d'un secteur qualifié (milieu nature, scolaire, d'entreprise, étranger, sportif, récréatif). Une aire protégée bien gérée constitue l'unique espoir de récupération pour une zone de montagne économiquement marginale avec des tendances à la désertification. Ce n'est que de cette façon que la plaie de notre temps, le chồmage, en particulier celui des jeunes intellectuels, pourra êtrecombattuen offrant uneidentité et de l'espoir a ux communautés intéressées. Des recherches scientifiques sont menées dans le parc pour mieux connaître le mode de vie des ours et des loups. Les moyens les plus sophistiqués (radiotélémétrie, phonolocalisation) sont utilisés pour érudier le comportement alimentaire de ces animaux, les couloirs de liaison entre les diverses zones... A cela s'ajoutent d'autres effers bénéfiques non quantifiables dus à la protection des ressources primaires: eau, 
air, fertilité des sols, stabilité du climat qui constituent des éléments essentiels à toute forme de vie et de développement.

Lemeilleurexemple de développement est constitué par le centre pilote de Civitella Alfedana, un village de 300 à 400 habitants situé à 1123 mètres d'altitude, abandonné pendant les années 1960, après l'exploitation «sauvage» des bois, le déclin de l'álevage transhumant et *sauvé par le loup». Il a été procédé à la revitalisation du centre historique avec naissance de nouvelles entreprises et retour de nombreux émigrés. Des jeunes gens dynamiques ont ouvert des hôtels, des pensions, des restaurants, d'autres sont devenusdes guides spécialisés pour des excursions à pied et à cheval. Le petit villagemoribond s'est remis à vivre. II peut offrir 500 lits grâce à un hôtel, 2 auberges, 1 auberge pour la jeunesse, de nombreuses chambres et maisons en location, 1 centre pour la visite des loups en semi-liberté, de nombreux sentiers nature, un centre culturel, uneagence de tourisme, desinitiativesd'animation et de promotion. Autre élément significatif, la banque artisanale est passée de quelques centaines de millions à presque 60 milliards de lires de dépôts. Les statistiques Bankitalia-Lstat ont révélé, à la surprise générale, que ce village est le premier centre italien pour l'épargne dépassant même les zones fortement industrialisées du Nord. Face à ce résultat, la banque locale a demandé et obtenu de reproduire sur ses chèques le petit ours, emblème du Pare National des Abruzzes. Un phénomène semblable se produit dans la zone Mainarde rattachée récemment à la zone $\mathrm{A}$ du Parc. Des villages condamnés ont pu revivre. Un bureau de zone du Parc a été créé, un centre de formation pour joueurs de Zampognne $e^{(1)}$ et un cours de qualification pour 40 jeunes locaux ont vu le jour. Des activités éducatives et touristiques se développent, un musée de la culture du Molise a ouvert ses portes, un centre de recherche scientifique destiné surtout à la faune est également crée.

\section{Perspectives d'avenir}

Voici vingt ans, l'Italie n'avait qu'l $1 \%$ de son territoireprotégé, elleen a actuellement $4 \%$. La campagne *Terre des Parcs Verts» lancée par WWF et par le comité Parcsen 1990, année des Parcs, a eu un rôle décisif. Après la phase de mise en marche laborieuse, le projet qui comprend les Abruzzer, le Gran Sasos, la Maiella et une partie des monts Sibillini concerne 300000 hectares. L'engagement tenace du Ministre de l'Environnement Valdo Spini qui a su en quelques mois remettre en mouvement le mécanisme mis en place par la loicadre fait qu'une nouvelle Italie des Pares esten train d'apparaitre d'une façon encore imparfaite, mais irréversible. Si l'objectif déclaréde $10 \%$ du territoire italien protégé est atteint, les visiteurs pourraient passer à 60 millions par an. Les emplois directs passeraient à au moins 10000 et ceux résultant de l'effet induit à plus de 50000 .

Autour du Parc National des Abruzzes un «systèmes de Parcs Nationaux du nom de ARVE est en train de se mettre en place. II s'agira alors d'un ensemble qui couvrira 600000 hectares et concernera toutes les valeurs naturelles plus importantes de l'Apennin Central. Cinq Régions seront alors intéressées: Abruzzes, Marches, Ombrie, Latium et Molise. Les Abruzzes seront une Région verte d'Europe (South European Park). L'impact économique sera alors de 20000 milliards de lires par an et le défi lancé à Camerino ${ }^{(14)}$ lors du congrès national du 28 au 30 octobre 1980 sera gagné et consacrera une splendide victoire pour les écologistes et les hommes de culture qui depuis des années se battent avec ténacité pour que la meilleure partie de l'Italie soit sauvée des risques qui la menaçaient et l'auraient déformée et dégradée à jamais. Comme l'a écrit G. Weeden, le emonde a besoin des sentiments suscités par les horizons inexplorés, les mystères des espaces sauvages. Il a besoin d'un lieu où les loups apparaissent à l'orée des bois lorsque descend le soir, parce qu'un milieu capable de produire un loup a un environnement sain, fort et parfaits.

$$
千
$$

\section{REEFERENCES}

(1) Le professeur Franco Tassi est actuellement directeur général des parcs taliens. Pendant des decennies il a combattu pour que le Parc des Abruzzes devenne une des azones vertesu les plus imponantes d'Europe. C'est grice a lui que nous avons pu obtenir les documents qui nous ont permis d'ecrire cet article. Nous tenons à le remercer vivement et publiquement ic:

(2) Les inondations quil le 5 et 6 novembre 1994 ont causé en Piémont la mort de dizaines de personnes et provoque des dégáts considerables en sont. un yivant exemple.

[3) Amedeo Postiginone. L'Amplamento der Parch Nazionah con particolati nterimenti al Parco d'Abruzzo, Rome, 1993.

19) Saint-Franços d Assises qui exprime son amou: pour loutes les créatures terivit dans l'un de ses récits i fiorerti l'histoire du loup de Gubbio

(4) Le Nomisma est un bureau d'études de Bologne dirigé par Romano Prodi dont les données sont generalement fiables.
La Zampogna est une sorte de cornemuse typique de ces régions.

(6) Camerino, petite ville des Marches 